Aportes a la educación no formal desde el abordaje de proyectos de acción social en la región Pacífico Central de Costa Rica María José Quesada Chávez

Extensión en red (9), e007, septiembre 2018

ISSN 1852-9569 | https://doi.org/10.24215/18529569e007

http://perio.unlp.edu.ar/ojs/index.php/extensionenred

FPyCS | Universidad Nacional de La Plata

La Plata | Buenos Aires | Argentina

\title{
Aportes a la educación no formal desde el abordaje de proyectos de acción social en la región Pacífico Central de Costa Rica
}

\author{
Contributions to Non-Formal Education from the Approach
}

of Social Action Projects in the Central Pacific Region, Costa Rica

\author{
María José Quesada Chávez \\ majosequesada@gmail.com \\ https://orcid.org/0000-0002-2901-2297 \\ Universidad de Costa Rica | Costa Rica
}

Resumen

En el artículo se presentan los resultados de una investigación realizada en 2017 que buscó discutir los aportes que realizan en el campo de la educación no formal los cursos desarrollados como parte de los proyectos de Acción Social de la Sede del Pacífico de la Universidad de Costa Rica.

La información se obtuvo por medio de un cuestionario a personas que participaron de las capacitaciones y de observaciones de campo realizadas por la investigadora. La discusión que se presenta es descriptiva con anotaciones cualitativas. Se determina que los conocimientos adquiridos han permitido a los participantes mejorar sus condiciones y su calidad de vida.

\section{Palabras clave}

educación no formal, proyecto, acción social, trabajo en comunidad

\section{Abstract}

The article presents the results of an investigation carried out in 2017 that aimed to discuss the contributions made in the field of non-formal education through courses developed as part of Social Action projects of the Pacific Campus of the University of Costa Rica. The information collected was obtained through a questionnaire addressed to people who have been part of the training offered

by the different projects as well as field observations made by the researcher. The discussion presented is descriptive with qualitative annotations. It is determined that the knowledge acquired has allowed participants improve their quality of life.

Keywords

non-formal education, project, social action, community work 


\title{
Introducción
}

La creación de espacios para la construcción del aprendizaje no formal es necesaria para permitirle a la población mejorar su calidad de vida. Dicha formación no solo resulta necesaria para las personas que no tuvieron acceso a los sistemas de educación formal durante su niñez o su adolescencia, sino también para los profesionales que requieren de actualización constante. Como afirma Catalina Turbay (2000):

\begin{abstract}
Los procesos o los caminos por excelencia que emplea la humanidad son la socialización y la educación; dos procesos interrelacionados a través de los cuales las sociedades humanas se aseguran que las nuevas generaciones hagan parte de ellas y contribuyan a su perpetuación y a su desarrollo. Así, la educación aparece como algo inherente a la especie humana a través de su historia con miras a garantizar la supervivencia del individuo y de la especie (p. 10).
\end{abstract}

Al respecto, Jorge Camors (2006), señala: «Podemos compartir con Jaume Trilla que la educación no formal se refiere a todas aquellas instituciones, actividades, medios, ámbitos de educación que, no siendo escolares, han sido creados expresamente para satisfacer determinados objetivos educativos» (p. 27).

Desde temprana edad, se disfruta del derecho humano a la educación por medio de sistemas de educación formal, tanto en el nivel preescolar, primario y secundario como durante la educación superior. En el caso de Costa Rica, el artículo 56 del Código de la Niñez y la Adolescencia (2008) dicta:

\footnotetext{
Las personas menores de edad tendrán el derecho de recibir educación orientada hacia el desarrollo de sus potencialidades. La preparación que se les ofrezca se dirigirá al ejercicio pleno de la ciudadanía y les inculcará el respeto por los derechos humanos, los valores culturales propios y el cuidado del ambiente natural, en un marco de paz y solidaridad (art. 56).
}

De acuerdo con Marcelo Morales (2009), la educación es una forma de transmitir el legado y la cultura unos a otros. Es por esta razón que no se les debe restar importancia a los modelos de aprendizaje no formal, toda vez que permiten reforzar, mejorar o adquirir nuevos conocimientos que no fueron abordados desde el sistema de educación formal. 
En reconocimiento a la labor realizada para promover la educación no formal desde los proyectos de Acción Social desarrollados por la Universidad de Costa Rica Sede del Pacífico, resulta importante anotar los siguientes aspectos:

a) Hasta la fecha, la Sede del Pacífico cuenta con veintidós proyectos de extensión docente, once de extensión cultural y cuatro de trabajos comunales. De estos 37 proyectos, diez realizan capacitaciones (el programa del adulto mayor, capacitaciones bilingües en inglés con fines específicos, etapa básica de artes integradas, educación continua, cursos libres, entre otros). Los cursos abarcan ramas tales como inglés, preparación física, artes plásticas, música, administración e informática.

b) Las personas que participan de estos proyectos proceden de diez comunidades: Orotina, Miramar, Esparza, Jacó, Isla Chira, Islita, Quepos, Costa de Pájaros, Cóbano y Puntarenas.

c) A lo largo de un año se logró la apertura de más de 200 cursos en el marco de dichos proyectos, lo que permite constatar el interés de la comunidad, pues siempre solicitan darle seguimiento al proceso iniciado.

Mediante el vínculo academia-sociedad, la acción social de la Universidad de Costa Rica en la Región Pacífico Central empodera a las comunidades vulnerables y a las poblaciones más olvidadas basándose en la ideología de la educación popular, con el fin de que potencien sus habilidades mediante procesos de instrucción no formal y puedan aspirar a lograr un mejor empleo o a establecer su propio emprendimiento, lo cual va a repercutir en el contexto socioeconómico en el que se desenvuelven diariamente.

El desarrollo de diferentes destrezas y habilidades les permite a las personas aspirar a diversos trabajos y optimizar notablemente sus condiciones de vida. Si bien en algunas regiones ya se habían realizado experiencias de educación no formal, debido a que el Instituto Nacional de Aprendizaje y la Universidad Estatal a Distancia en algún momento habían brindado talleres, la solicitud siempre ha sido que no se desarrolle una sola capacitación aislada, sino que sea parte de un proceso, y hacia esto se ha ido trabajando con la apertura de cursos libres, cortos y gratuitos en la provincia de Puntarenas.

Al convertirse estas prácticas en espacios organizados, sistémicos y con fines educativos - sea para abordar contenidos académicos, como el aprendizaje de una segunda lengua, o con fines artísticos, como incursionar en el mundo de la música o de las artes plásticasse crearon oportunidades que enriquecieron a la población, tanto a nivel teórico como cultural. Los cursos cortos o programas modulares que se han ofrecido a lo largo de los años, por medio del trabajo realizado desde la acción social o la extensión universitaria, en ocasiones se han desvalorizado y no se les ha dado el apoyo para que el proceso continúe. 
De este modo, se deja de lado la realidad que viven las comunidades de la zona para las cuales, en muchos casos, el acceso a este tipo de cursos resultaría inalcanzable si lo tuvieran que matricular en una institución privada. Por lo anterior, con el presente trabajo investigativo se pretende crear conciencia sobre la importancia de desarrollar actividades de educación no formal por medio de los proyectos de acción social, no solo para la población que recibe el curso sino para la universidad, ya que es en este diálogo directo en el contexto de cada realidad que se logra un verdadero acercamiento y se enriquece el vínculo universidad-sociedad en la Universidad de Costa Rica.

\section{Marco teórico}

La educación no formal en el contexto de la acción social:

definición y características

La educación popular es una corriente de pensamiento que tiene como fundamento partir de los intereses de los sectores populares con el fin de que se logre, mediante la educación, una transformación social en beneficio de los pobladores según las necesidades que posean.

De la mano del concepto de educación popular sobresale el de educación no formal, entendida como

toda actividad educativa e instructiva estructurada y sistemática, de duración relativamente breve, por medio de la cual las entidades patrocinadoras se proponen lograr modificaciones concretas de la conducta de grupos de población bastante diferenciados (Pastor, 2001, p. 526).

Como contraposición a la educación no formal, es necesario definir la educación formal, que significa:

[...] el «sistema educativo» jerarquizado, estructurado, cronológicamente graduado, que va desde la escuela primaria hasta la universidad e incluye, además de los estudios académicos generales, una variedad de programas especializados e instituciones para la formación profesional y técnica a tiempo completo (Pastor, 2001, p. 527). 
De esta manera, la educación no formal desarrollada mediante los proyectos de acción social parte del estudio de requerimientos de poblaciones vulnerables con el fin de llenar brechas educativas o de reforzar lo que se recibió mediante la educación formal. En algunos casos, de acuerdo con la realidad de estos grupos, lo no formal se convierte en la única forma de instrucción que alcanzarán en la vida.

Debe resaltarse, entonces, la diferencia entre ambos sistemas de educación:

La educación formal y la educación informal son los dos campos que operan en lo social, y es, fundamentalmente, por la intervención del Estado pero también de la sociedad civil organizada que se empieza a dar forma a este núcleo de informalidad (Rodríguez, 2009, p. 158).

De esta forma, proyectos destacados en el área de extensión docente tienen como característica que el mismo profesor responsable del proyecto ejecute el proceso educativo o bien contrate a un profesional especializado que brinde dicha capacitación, tal como lo resalta Rosa María Torres (2009):

Luego tenemos la educación no formal, donde existe en efecto alguien que hace de educador y que tiene la intención de enseñar por fuera del sistema formal: enseñar a manejar, a bailar, a usar la computadora, etc. La educación no formal ha estado siempre ahí, como complemento de la educación formal (pp. 165-166).

Es por medio de la educación que se le permite a un grupo de personas cambiar y mejorar sus condiciones de vida, lo que debe verse como una herramienta que abre las puertas a la superación y al mejoramiento constante.

[...] es un proceso dinámico. Las acciones educativas se conciben en procesos dinámicos y necesariamente flexibles en sus modalidades, estructuras, contenidos y métodos. La educación es un proceso innovador. Se trata de buscar nuevas formas que satisfagan eficaz, adecuada y económicamente las necesidades educativas que se generan en el presente y, especialmente, en el futuro inmediato. Los principios de la educación permanente exigen innovaciones reales y profundas en el campo de las acciones educativas (Tünnermann Bernheim, 2010, p. 24).

En el caso de la educación no formal, sus propuestas deben ser mayoritariamente innovadoras, ya que no se enmarcan dentro del sistema educativo tradicional. Además, deben pensarse estrategias que permitan captar el interés de la comunidad y construir 
juntos la esperanza de poderse superar en el proceso educativo. Lorenzo García Arieto Marta Ruiz Corbella y Miriam García Blanco (2009), concuerdan con Carlos Tünnermann Bernheim (2010) al afirmar que, en este tipo de procesos, debe transmitirse o contagiarse un sentir dinámico, ya que muchas comunidades vulnerables viven sin interés, dormidas en el letargo del abandono y la desesperanza.

En definitiva, la educación es un proceso permanente en el desarrollo de todo individuo, es proyecto, tarea que debe estar presente a lo largo de la vida, que permite ordenar las distintas etapas, preparar las transiciones, diversificar y valorizar las trayectorias vitales de cada sujeto logrando que llegue a ser una persona única, capaz de afrontar su vida de modo autónomo en cada uno de los estadios vitales (García Arieto y otros, 2009, p. 67).

En todo proceso educativo existen retos. El hecho de carecer de una estructura administrativa y curricular organizada o preestablecida dificulta en algunas ocasiones los procesos de educación no formal. Sin embargo, todos estos obstáculos deben vencerse, ya que como afirma Jorge Grandi (2009):

La educación siempre está presente en todas las convocatorias, los análisis y los proyectos que aspiran a promover cambios y a enfrentar los desafíos de las sociedades en procura de una mejora, del fomento, del desarrollo de las personas y de las colectividades, de acuerdo a los problemas y las necesidades de cada época (p. 17).

Por su parte, Manuel Chacón-Ortiz (2015), retomando a Gonzalo Vázquez (1998), asegura que la educación no formal

\footnotetext{
[debe ser] un proceso educativo planificado, que no necesariamente lleva la consecución de un título o grado académico, con una duración y unos objetivos definidos, desarrollado por instituciones y por organizaciones de ámbitos diversos, estructurado en sus acciones, sus contenidos y sus métodos. Usualmente, además, visto como toda aquella educación que sucede, bajo estos estándares, fuera de las aulas (p. 25).
}

Es una realidad que se tengan que buscar espacios comunales en los que se puedan desarrollar las lecciones. Además, no hay una estructura fija o establecida que imponga normas y las reglas se construyen en conjunto con la comunidad. Al tomarse como base la legislación en la materia, se tiene que a la educación no formal se le añade un valor adicional: se trata de procesos que se plantean para cubrir una necesidad educativa de un grupo social. 
La educación no formal, en el marco de una cultura del aprendizaje y a lo largo de toda la vida, comprenderá todas aquellas actividades, medios y ámbitos de la educación que se desarrollan fuera de la educación formal, dirigidos a personas de cualquier edad, que tienen valor educativo en sí mismos y que han sido organizados expresamente para satisfacer determinados objetivos educativos en diversos ámbitos de la vida social, la capacitación laboral, la promoción comunitaria, la animación sociocultural, el mejoramiento de las condiciones de vida, la educación artística, tecnológica, lúdica, deportiva, entre otros (Morales, 2009, p. 92).

De esta manera, es importante profundizar en una conciencia capaz de crear un deseo por mejorar, por tomar un impulso y por vencer las barreras que han tenido a estas poblaciones en estado de vulnerabilidad por años; en algunos casos desde que nacieron. García Arieto, Ruiz Corbella y García Blanco (2009) afirman que la educación es un proceso de toda la vida, no tiene un culmen y debe proponerse, en primera instancia, colaborar para que cada persona pueda integrarse a la sociedad en la que se desarrolla. Debe aportar los requerimientos necesarios para poder «ser, conocer, hacer y convivir en contextos cada vez más cambiantes y dinámicos» (p. 71).

A la educación no formal se le deben reconocer el valor y el potencial que promueve para el desarrollo social. Según Morales (2009), dada la diversidad de experiencias en esta área, se les debe dar un fuerte apoyo para que se conviertan en procesos de calidad que favorezcan el crecimiento educativo. Además, es importante considerar que mediante la educación no formal es posible lograr un acercamiento y crear un vínculo para que procesos de educación formal se puedan culminar. Por esta razón, si se pretende lograr un cambio en la sociedad, y en el país en general, es necesario promover estas iniciativas en las comunidades.

La educación no formal es una importante herramienta de transformación social. Reconocida mundialmente por la UNESCO y por muchas otras instituciones, se ha convertido en un recurso habitual en ámbitos como la educación continua, organizaciones no gubernamentales, grupos culturales, entre muchos. Los alcances de la educación no formal son reconocidos como claves, tanto para el ejercicio de equidad $\mathrm{y}$ de justicia social en situaciones de vulnerabilidad, como en circunstancias que complementan el pleno desarrollo de las facultades humanas en el marco de una sociedad de información, donde las personas constantemente están en la búsqueda de nuevos conocimientos, los procesos educativos no formales se han convertido en un recurso común de la sociedad (Chacón-Ortiz, 2015, p. 32). 
Aspectos metodológicos relacionados con la educación no formal:

sujetos participantes, estrategias y evaluación

La educación no formal no puede enmarcarse en una estructura administrativa definida, ya que va a depender de las condiciones y las características propias del contexto en el que se desarrolle. Al tratar de caracterizar los procesos que se llevan a cabo en este ámbito, puede decirse que

\begin{abstract}
Las instituciones de formación social y cultural son centros que desarrollan una serie de programas educativos fuera del ámbito formal, esto quiere decir que dichos programas se llevan a cabo fuera de la legislación del sistema educativo regularizado, donde se proponen juegos y otras actividades [...] que no se pueden encontrar en otros contextos, y proporcionarán, entre otras cosas, una expansión y una mejora de las relaciones interpersonales (Torres Martín \& Pareja Fernández de la Reguera, 2007, p. 24).
\end{abstract}

En estos procesos, las evaluaciones son totalmente necesarias. Sin embargo, en esta modalidad educativa se entra en un debate, debido a que, en ocasiones, la población se resiste a adquirir un compromiso y decide abandonar las clases antes de someterse a los diversos criterios evaluativos de los cursos, los cuales no siempre brindan un certificado.

\begin{abstract}
Al desarrollar programas educativos no formales, no interesa o no se le da la importancia suficiente a la evaluación. En muchos casos, esto se atribuye a las cualidades de los programas, dado que la educación no formal no conlleva necesariamente a titulaciones, menos una titulación del sistema educativo formal, por ello, lamentablemente, la evaluación en algunos casos pasa a un segundo plano (Chacón-Ortiz, 2015, p. 22).
\end{abstract}

Entonces, más que una evaluación de los aprendizajes, el autor argumenta que lo que se requiere es de una evaluación del proceso o del desarrollo de los programas de educación no formal.

La evaluación del impacto o productos está vinculada con el ámbito y los resultados esperados en las condiciones de vida. Evaluación de impacto o del producto: establecer evidencia entre el programa y su relación con los resultados. Lo difícil de esta evaluación es establecer una relación causal entre los resultados observados y las actividades de un programa. 
Finalmente, dentro de estas categorías más empleadas, se debe mencionar la evaluación de la planificación, es decir, de las acciones, de la forma en que estas desarrollan. Medir si las acciones están desarrollándose en forma adecuada, de acuerdo con principios éticos, mecanismos de control, regulación y registro, tal como han sido estructurados en el marco de la evaluación (Chacón-Ortiz, 2015, p. 30).

La metodología educativa de estos cursos supone procesos flexibles que permiten, a la vez, dinamizar el trabajo que se realiza en el seno de las comunidades. En este sentido, «la educación no formal ofrece una abundante y dispar perspectiva, no solo en cuanto a sus funciones educativas, sino también con relación a las instituciones, agentes, métodos y procedimientos que forman parte de esta particular modalidad educativa» (Torres Martín \& Pareja Fernández de la Reguera, 2007, p. 36).

Asimismo, su administración conlleva, como señala Manuel Luján Ferrer (2010) «una compleja gama de fases determinadas por la planificación, la organización, la ejecución, el monitoreo y la evaluación de las actividades humanas que permiten brindar servicios y la producción de bienes de manera eficiente y eficaz» (p. 107). Debe pensarse, entonces, en las características de cada grupo para poder plantear así cada acción, tener un monitoreo constante y una evaluación que permitan valorar aciertos y situaciones a mejorar.

\section{La educación no formal como proceso educativo que favorece a la comunidad en general}

Las comunidades en condiciones de vulnerabilidad necesitan de manera imperativa contar con opciones educativas que les permitan a sus pobladores tener acceso a opciones para mejorar sus condiciones sociales y culturales.

La programación de cursos específicos para la educación no formal está justificada por la cada vez mayor demanda social, es necesario formular programas que den una respuesta satisfactoria para mejorar la calidad de estas enseñanzas, no se descubre nada nuevo al decir que estas enseñanzas están frecuentemente impartidas por aficionados/as con conocimientos superficiales sobre los cursos que dirigen, especialmente si se refieren a la enseñanza del arte, donde se valora positivamente la figura del/a autodidacta que con demasiada frecuencia esconde personajes de dudosa formación, casos que no se producen en otras áreas del conocimiento donde el intrusismo es condenado con severidad. Es necesario encauzar las acciones, proponer 
estrategias, para un futuro mejor. Es hora de transformaciones y un momento adecuado para validar conocimientos, de asumir cada cual su papel (Abad Tejerina, 2002, p. 421).

Es característico de las poblaciones en riesgo social no tener acceso a los sistemas de educación formal, ya que por su edad o por sus condiciones económicas deben trabajar y no les queda tiempo para el estudio; ello, sumado a la falta de compromiso y a la desmotivación en la que se encuentran inmersos. Son poblaciones olvidadas, pero están deseosas de poder contar con el apoyo para prepararse y para generar mejores condiciones de vida para sus familias.

La educación no es algo reservado a algunos pocos, sino un derecho de todos; se trata de un deber y de un derecho específico de todo ser humano, dirigido a desarrollar todas y cada una de sus posibilidades. Sin duda,

[...] la educación y la formación serán, más que nunca, los principales vectores de la identificación, la pertenencia y la promoción social. A través de la educación y de la formación, adquiridas en el sistema educativo institucional, en la empresa, o de una forma más informal, los individuos serán los dueños de su destino y garantizarán su desarrollo (García Arieto y otros, 2009, p. 150).

\section{Metodología}

En las diferentes capacitaciones brindadas mediante la ejecución de diez proyectos de Acción Social se realizaron encuestas con el fin de identificar el grado de satisfacción de los participantes, así como los resultados obtenidos en las diferentes áreas del conocimiento abarcadas. Se trató de un estudio de campo en aulas de instituciones del Ministerio de Educación Pública y en salones comunales o espacios municipales.

La población en estudio estuvo conformada, en su mayoría, por personas en condiciones de vulnerabilidad, tales como adultos y adultas mayores, mujeres jefas de hogar, niños y niñas, y jóvenes. Esto es característico en procesos de educación no formal, ya que en ellos participan personas de todas las edades y géneros, sea cual sea su condición social:

[...] a veces pensamos que la educación se restringe a algunas edades, a algunas etapas de la vida. La ENF [educación no formal] tiene para aportar en todas las etapas y en todas las situaciones, toda persona tiene derecho a participar en propuestas educativas que le ayuden a mejorar su vida en el sentido que desee (Morales, 2009, p. 93). 
En el estudio, participaron alrededor de 100 estudiantes y ocho docentes responsables de proyectos de acción social. Para obtener la información, se aplicó un instrumento a los/las participantes de las capacitaciones que consistió en un cuestionario con preguntas cerradas en el que debían indicar, según la escala, el grado de cumplimiento o de satisfacción con las temáticas desarrolladas (ver Anexo). De igual forma, durante el desarrollo de los proyectos se trabajó con la observación no participativa, a fin de valorar la incidencia que se logra para satisfacer las necesidades de capacitación de las diferentes poblaciones analizadas.

El instrumento fue distribuido de manera impresa, se completaba en menos de diez minutos y, en muchos casos, el/la docente a cargo colaboró en su aplicación.

\title{
Resultados
}

Los cursos cortos desarrollados en las diferentes comunidades tienen como propósito abordar diferentes áreas del conocimiento para satisfacer las necesidades educativas de la población, lo cual es uno de los principales propósitos de la educación no formal. Al respecto, Chacón-Ortiz (2015) indica:

\begin{abstract}
La educación no formal es un espacio educativo en crecimiento. Esta modalidad surge como opción para llenar algunos vacíos o necesidades formativas que los sistemas formales no han podido o no están en obligación de cumplir. Entre estos se encuentran algunas modalidades de la enseñanza técnica, círculos de estudio, actividades extracurriculares y fuera del aula. La Organización de las Naciones Unidas para la Educación, UNESCO (Hoppers, 2006) reconoce y promueve la educación no formal como herramienta de empoderamiento y de transformación social (p. 22).
\end{abstract}

Al consultar a las/los participantes sobre el grado de satisfacción, comparando las expectativas que tenían antes de matricularse y luego de culminar la capacitación, 80 por ciento manifestó encontrarse muy de acuerdo con el proceso formativo recibido, ya que la información suministrada, tal como lo menciona el autor, les permitió empoderarse y fungir como pieza de transformación social.

Antes de ofrecer una capacitación en cualquiera de las áreas, la/el docente debe realizar una investigación sobre las necesidades reales de la comunidad con el fin de proponer una oferta educativa acorde a la realidad que se vive en cada contexto. Como señala Camors (2009), «el trabajo educativo en este campo debe estar sustentado en orientaciones, en 
ideas previas, en estudiar la situación de los sujetos y sus contextos a los efectos de diseñar una estrategia educativa» (p. 33). De igual manera, Luján Ferrer (2010) afirma: «Los diagnósticos participativos son procesos esenciales en la administración de la educación no formal que permiten adecuar sus procesos al contexto sociocultural y a las condiciones particulares de las poblaciones beneficiarias de sus programas» (p. 117).

En el caso del presente estudio, cuando se le consultó a la comunidad sobre la pertinencia de la capacitación brindada, 75 por ciento coincidió en que los objetivos estaban estrechamente relacionados con una necesidad que poseían antes de recibir la instrucción.

Es imposible hacer una generalización de las características para poder establecer un solo modelo curricular a seguir en la educación no formal, toda vez que

Cada grupo social dispone de su propia cultura que le hace ser peculiar y distinto de otros grupos, por el conjunto de comportamientos, de actitudes y de valores que conforman su modo de vida y su propia identidad. Cultura que entendemos como todo aquel universo simbólico que el hombre ha elaborado, transformando el mundo físico y natural. Esta cultura, que representa el aspecto dinámico de la estructura social, se acepta, se comparte, se defiende y se transmite de unas generaciones a otras. Transmisión que supone aprendizaje, dado que la cultura no se hereda en el sentido biológico del término. Este aprendizaje trasciende al realizado en cualquier institución educativa, aunque esta extienda cada vez más su acción, verticalmente (ampliación de los años de enseñanza obligatoria, acceso mayoritario los niveles de enseñanza secundaria y superior), como horizontalmente (acceso a la educación de toda la población) (García Arieto y otros, 2009, p. 177).

Luego de realizado el análisis de necesidades se pueden tomar decisiones importantes con el fin de ofrecer un proceso educativo acorde con las necesidades de formación.

El análisis de necesidades previas no nos exime de ese momento tan necesario al comienzo de un proceso formativo: el ajuste formativo. Es muy probable que las primeras sesiones se dediquen a la recogida de las expectativas y al nivel de conocimiento y de práctica del grupo permitiéndonos ajustar nuestro programa a estos aspectos. Este proceso es complejo (programas muy cerrados que no permiten cambios, acuerdos con profesorados, duración determinada). Aun así, es muy posible que podamos centrar los objetivos, los contenidos, el método y las normas de funcionamiento a las necesidades del grupo con el que vamos a trabajar (Lamata \& Domínguez, 2003, p. 128). 
El diseño y el desarrollo metodológico de la educación no formal se constituye en un reto para el educador y la educadora, ya que en los ambientes en los que se desarrolla se encuentran poblaciones muy diversas y con particularidades muy específicas. Se debe tomar en cuenta que, en algunos casos, las personas no saben ni leer ni escribir, por lo que debe partirse, entonces, de cada una de esas realidades.

En este sentido, se concuerda completamente con lo que Morales (2009) apunta cuando se refiere a las metodologías utilizadas en el sistema de educación no formal:

\begin{abstract}
Desarrollar propuestas educativas de calidad exige profesionales capaces de diseñarlas y de llevarlas adelante. La ENF, por su diversa riqueza, exige educadores capaces de leer los contextos en los que se producen las prácticas, seleccionar contenidos, buscar metodologías adecuadas para enseñarlos, mostrarlos, donde muchas veces el punto de partida es el intento por generar el deseo de aprender (algo) en el otro y el convencimiento de que puede hacerlo. La no existencia de currículos preestablecidos, la diversidad de marcos institucionales, la variedad de objetivos de las distintas propuestas educativas, el movimiento de cambios en el que venimos inmersos como sociedad, hacen ver la necesidad de profesionales capaces de pensar pedagógicamente desde escenarios radicalmente diferentes (p. 91).
\end{abstract}

Además, es importante reconocer que los procesos de educación no formal permiten manejar un rango de flexibilidad que puede tomarse como ventaja o como desventaja. En el caso de los cursos desarrollados en el marco de los proyectos de Acción Social se convirtió en una ventaja, ya que de esta forma se pudieron diseñar cursos cortos de dos semanas, bimensuales, trimestrales y/o semestrales. Como indica Camors (2009): «La flexibilidad en el manejo de los tiempos es importante en función de las posibilidades de la población, siempre buscando adecuar la propuesta a las personas» (p. 34). ChacónOrtiz (2015), por su parte, también señala que «la particularidad esencial de la educación no formal es su flexibilidad y su adaptabilidad a los distintos contextos sociales» (p. 22).

Al tratarse de experiencias y de poblaciones nuevas para muchos de los/las docentes, se debe reconocer que aún quedan aspectos por mejorar. De los entrevistados, 90 por ciento indicó que la metodología y el cumplimiento de los objetivos fueron satisfactorios, mientras que solo 10 por ciento señaló estar en desacuerdo. Otro aspecto en desventaja es el hecho de que a las educadoras y los educadores se los prepara a nivel universitario para desarrollar su profesión dentro del sistema educativo formal, por lo que hay una falta de capacitación para ejercer en el campo de la informalidad. 
[La educación no formal] requiere de un perfil de educador específico. Se requiere de un profesional de la educación preparado para trabajar objetivos y contenidos educativos, cuya amplitud y diversidad dependerá de las necesidades y los intereses de los participantes. Se requiere flexibilidad para desarrollar su trabajo en espacios y en momentos muchas veces asediados por circunstancias imprevistas o afectados por elementos del contexto social, comunitario o de los propios participantes (Camors, 2009, p. 29).

De esta forma, al trabajar bajo la modalidad de educación no formal, cuando se evalúa el curso no debe pensarse únicamente en el desempeño docente y en la metodología aplicada, sino que, tal y como señala Chacón-Ortiz (2015), debe evaluarse el impacto social logrado.

La evaluación y el desarrollo de programas se miden tanto por su impacto en poblaciones o en grupos, como por el proceso educativo en sí mismo; por ejemplo, el alcance que tiene este en una comunidad, la cantidad de personas participantes y la cualidad de los grupos que asisten pueden ser indicadores igualmente importantes. Para cualquiera de estas dos, es necesario el establecimiento de objetivos que permitan ver hacia dónde se quiere llegar (p. 23).

Como puede observarse en el siguiente gráfico, el estudio realizado refleja que 70 por ciento de los participantes manifestó estar muy de acuerdo con el beneficio o con el impacto social alcanzado; el otro 30 por ciento indicó estar de acuerdo.

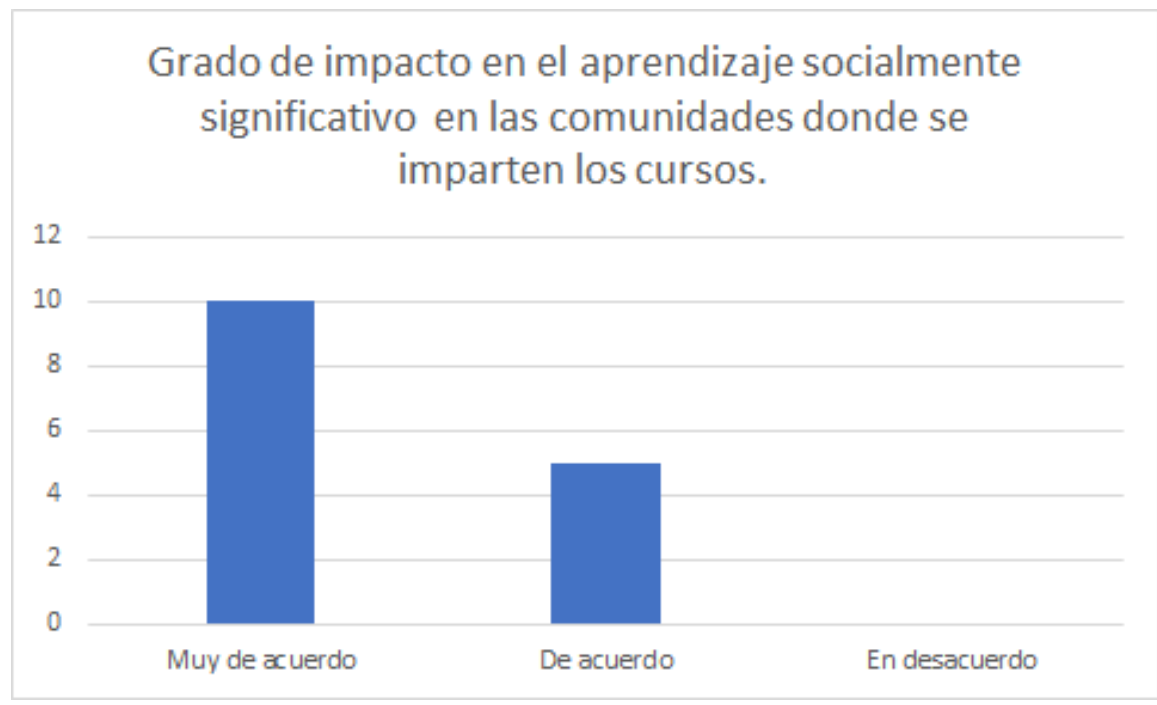

Opinión de los participantes sobre la aplicabilidad de los aprendizajes adquiridos en el curso (Fuente: elaboración propia) 
Indudablemente, si una comunidad quiere superarse, no hay más opción que contar con educación de calidad. Como se ha desarrollado en la discusión anterior, no se puede tener como única opción los sistemas de educación formal conocidos, sino que la educación no formal se convierte en la aliada protagónica para permitir a las personas cambiar y mejorar sus condiciones de vida.

Es patente la relación existente entre el desarrollo económico y social de un país y su sistema educativo. Los países desarrollados son conscientes de la importancia que la educación tiene para el progreso de su economía y de su bienestar social. Esta convicción es la que genera mayores inversiones proporcionales a su producto interno bruto (PIB). Es decir, a mayor desarrollo de un país, mayores esfuerzos por adecuar la educación a las complejas y múltiples necesidades de las sociedades tecnológicas, ya que el motor del progreso se encuentra en la cualificación profesional de los ciudadanos, que son los que realmente sustentarán las estructuras sociales y políticas generadoras de este proceso. La educación se convierte, así, en una de las dimensiones del desarrollo global, al igual que la economía o la política (García Arieto y otros, 2009, p. 179).

En tanto, cuando se realiza la observación no participativa como responsable de la investigación se logra constatar la gran diversidad de asistentes en los diferentes cursos de música, de emprendedurismo, de inglés y de arte. Las y los participantes provienen de comunidades en riesgo social: son niños y niñas, jóvenes y mujeres jefas de hogar que han abandonado el sistema educativo formal debido a que no contaron con el apoyo de sus padres, o bien porque debían aportar a la generación de ingresos para ayudar a su familia. Los y las estudiantes de estos cursos tienen realidades muy fuertes y características muy particulares; incluso, se encontraron algunos casos de discapacidad física o mental.

Muchas veces, se pensó que estos cursos podían ser impartidos en cualquier momento del día. Sin embargo, por las características socioeconómicas de la población, se comprobó que debían dictarse en horarios vespertinos o nocturnos, o bien los sábados. En la mayoría, se ofreció una instrucción gratuita, ya que cuando se intentaba abrir cursos pagos, aunque fuera a un bajo costo, no se alcanzaba la matrícula.

Sin duda alguna, todos los cursos desarrollados siguieron la ideología de la educación popular. Se asumió al individuo como un ser capaz de lograr la transformación social, de luchar por mejorar su comunidad, como un ser autónomo de quien se espera que pueda lograr un mejoramiento de su calidad de vida y de la de su núcleo familiar, con herramientas y con conocimientos construidos conjuntamente. 
Las y los estudiantes coinciden en que los cursos deben ser parte de un proceso, no capacitaciones aisladas o experimentos sociales mediante los cuales la academia llega, toma la información que requiere y los deja en el olvido. Por lo contrario, solicitan que la instrucción sea, incluso, organizada en módulos que les vayan dando titulaciones de acuerdo al nivel alcanzado.

\section{Conclusiones}

La educación es un componente social, por lo cual es imposible abordarlo sin antes efectuar un análisis de la sociedad en la que se va a desarrollar el proceso educativo. Por eso, es posible encontrar diferentes contenidos y objetivos para una misma temática en diversos contextos sociales.

La educación no formal se convierte en un componente importante para las poblaciones vulnerables dado que, para la gran mayoría, constituye la única forma de tener un acercamiento a la educación, a herramientas de inclusión y de empoderamiento, ya que, por su realidad social o económica, no les fue posible ser parte del sistema de educación formal.

Este tipo de educación debe romper paradigmas en las comunidades donde, muchas veces, su acceso a oportunidades educativas se frustrado dado el fracaso escolar, económico y social que viven. Al respecto, Jorge Balán (2009) indica: «Las oportunidades de aprendizaje continuo se han ampliado notoriamente gracias a la revolución en las tecnologías de información y comunicación, que han alterado significativamente qué y cómo se aprende tanto dentro como fuera de la escuela» (p. 39). De esta forma, la educación para adultos se ha relacionado con el hecho de propiciar un espacio donde se le permita al individuo promover transformaciones sociales.

Recientemente se han identificado, como espacios emergentes de la educación no formal, los grupos artísticos, musicales y culturales que atienden poblaciones diversas tales como personas adultas mayores, jóvenes, niñez, personas con necesidades especiales. En una sociedad donde la información es el elemento que articula cada día las formas de producción y de creación, es de esperar que la gente busque cursos y espacios de formación que le sirvan para mejorar sus condiciones de vida (ChacónOrtiz, 2015, p. 22). 
Los proyectos de acción social encargados de desarrollar procesos de educación no formal en las comunidades no deben olvidar la realidad social de exclusión, de injusticias, de vulnerabilidad y de violencia de los y las habitantes. Antes bien, deben analizar y potencializar las habilidades y las necesidades que se tengan a fin de canalizarlas y de impulsarlas a través del proceso educativo que se desarrolle.

Esta educación no formal, tal y como su nombre lo plantea, posee una gran ventaja: la libertad para plantear y para construir procesos de enseñanza acordes con la realidad y las necesidades de las y los pobladores. Por tal razón, está llamada a romper los esquemas tradicionales, mismos que han dejado grandes vacíos que deben llenarse a través de los procesos que se desarrollan producto del trabajo en comunidad.

La educación no formal en razón de su naturaleza, ámbitos de acción, poblaciones que atiende y modalidades es una opción socioeducativa para el mejoramiento de las condiciones de vida de los grupos sociales, en correspondencia con sus necesidades y sus expectativas (Luján Ferrer, 2010, p. 116).

Con la educación se hace posible la erradicación o el combate de los diferentes problemas sociales que carcomen a las comunidades del país. García Arieto, Ruiz Corbella y García Blanco (2009) indican que si lo que se quiere es lograr la modernización de la sociedad se debe potenciar una educación que permita la formación de hombres y de mujeres creativos/as, libres e innovadores/as.

Lo anterior debe relacionarse directamente con los retos que debe superar la educación no formal, los cuales se podrían resumir en tres tipos. El primero, se refiere a la necesidad de formación de instructores e instructoras con una visión social completa y real de la sociedad donde se desarrolla el proceso educativo que pretende alguna transformación.

El segundo, al requerimiento primordial de realizar una investigación de la realidad comunitaria antes de plantear cualquier proyecto de acción social relacionado con procesos que conlleven el desarrollo de la educación no formal. En ningún momento un proyecto puede surgir producto de un sentimiento personal del/la docente o como una forma de hacer caridad. La educación no formal tiene $-\mathrm{y}$ ha tenido-éxito y apertura en comunidades vulnerables debido a que su principal característica es la inclusión y la creación de espacios para la superación personal, sean cuales sean las condiciones de vida. 
La ENF puede ser efectiva, particularmente, donde el sistema formal es incapaz de cubrir las necesidades educativas de aquellos cuyos patrones de vida no coinciden con sus preconcepciones; o donde aquellos que han abandonado la escuela no están en capacidad o no se sienten inclinados a volver a unirse al sistema convencional (Dave, Ranaweera \& Sutton, 1990, p. 36).

El tercer reto se refiere a un desafío logístico el cual se relaciona con el hecho de que este tipo de educación debe ser gratuita dado el contexto en el que se desarrolla. No obstante, en algunas ocasiones, se experimenta una falta de compromiso por parte de los y las estudiantes, ya que al ser gratuitos no hay ningún remordimiento en abandonarlos. No obstante, al llevar opciones educativas por medio de procesos de educación no formal se devuelven la esperanza y los deseos de superación, no solo a adultos y adultas, sino también a las nuevas generaciones, lo que permitirá, deseablemente, romper con el círculo de pobreza en que se han visto inmersos y, a su vez, contribuir a la formación de ciudadanos y de ciudadanas libres y críticos/as, y a que no sean más esa población en desventaja, ya que se poseen las herramientas que permiten superarlas. 


\section{Cuestionario sobre Educación No Formal}

Dirigido a los estudiantes y padres de familia de la población de cursos libres. Su información es confidencial y será de gran apoyo para la continuidad de este tipo de cursos. Responda, por favor, a todas las preguntas.

1. ¿Estaría dispuesto a darle continuidad al curso en caso que se requiera cobrar por el costo de la institución un monto módico?
( ) Sí
( ) No

2. ¿Qué otro tipo de capacitación considera usted que se requiere llevar a cabo en su comunidad?

3. ¿Qué beneficios para la comunidad pueden presentarse al recibir este tipo de capacitación?

4. ¿Qué inconvenientes para la comunidad pueden presentarse al recibir este tipo de capacitación?

5. Además de la Universidad de Costa Rica alguna otra institución ha brindado o brinda apoyo para capacitar en su comunidad
( ) No
( ) Sí, cuáles:

6. ¿Cuál fue su principal motivación para llevar este tipo de cursos?

7. ¿Considera usted importante que se le dé un seguimiento al curso?

( ) Sí ( ) No

8. ¿Estaría anuente a volver a matricular otro curso en esta misma área?
( ) Sí
( ) No

9. ¿Existe un clima dentro de su comunidad donde los habitantes se organizan para promover espacios continuos de formación y capacitación?
( ) Sí
( ) No

10. ¿Qué tipo de población dentro de su comunidad considera usted que es lo que más requiere de este tipo de capacitaciones?
( ) Adultos
( ) Jóvenes
( ) Niños
( ) Mujeres jefas de hogar
( ) Adultos mayores 
Elija, por favor, la respuesta más apropiada para cada pregunta, marcando con una equis la casilla correspondiente.

\begin{tabular}{|l|l|l|l|l|l|}
\hline & $\begin{array}{c}\text { Muy de } \\
\text { acuerdo }\end{array}$ & $\begin{array}{c}\text { En } \\
\text { desacuerdo }\end{array}$ & Indeciso & $\begin{array}{c}\text { De } \\
\text { acuerdo }\end{array}$ & $\begin{array}{c}\text { Muy de } \\
\text { acuerdo }\end{array}$ \\
\hline $\begin{array}{l}\text { 1. La capacitación recibida a } \\
\text { ayudado a mejorar su condición } \\
\text { socioeconómica. }\end{array}$ & & & & & \\
\hline $\begin{array}{l}\text { 2. Los contenidos desarrollados } \\
\text { están de acuerdo con la } \\
\text { necesidad que se tenía en } \\
\text { cuanto a capacitación. }\end{array}$ & & & & & \\
\hline $\begin{array}{l}\text { 3. La evaluación desarrollada } \\
\text { estuvo acorde con la meta del } \\
\text { curso. }\end{array}$ & & & & & \\
\hline $\begin{array}{l}\text { 4. El tipo de capacitación } \\
\text { brindada satisfizo sus } \\
\text { expectativas. }\end{array}$ & & & & & \\
\hline $\begin{array}{l}\text { 5. El docente cumplió con el } \\
\text { logro del objetivo del curso } \\
\text { propuesto. }\end{array}$ & & & & & \\
\hline $\begin{array}{l}\text { 6. Con el curso se promovió un } \\
\text { aprendizaje socialmente } \\
\text { significativo y apropiado a las } \\
\text { realidades culturales de su } \\
\text { comunidad. }\end{array}$ & & & & & \\
\hline
\end{tabular}




\section{Referencias}

Abad Tejerina, M. J. (2002). Educación artística en el ámbito de la educación no formal. Arte, individuo y sociedad, (s/n), 421-426. Recuperado de https://revistas.ucm.es/index.php/ARIS/article/view/ARIS0202110421A

Balán, J. (2009). Las políticas de educación no formal y de educación de jóvenes y adultos desde la perspectiva del aprendizaje a lo largo de toda la vida. En M. Morales (Comp.), Aportes para la elaboración de propuestas de políticas educativas (pp. 39-64). Recuperado de http://www.oei.es/pdf2/aportes_educacion_no_formaluruguay.pdf

Camors, J. (2006). Educación no formal: Fundamentos para una política educativa. Montevideo, Uruguay: Ministerio de Educación y Cultura. Recuperado de http://educacion.mec.gub.uy/boletin/publinoformal/publicacion_\%20ENF.pdf

Camors, J. (2009). Educación No Formal. Política educativa del MEC 2005-2009. En M. Morales (Comp.), Aportes para la Elaboración de Propuestas de Políticas Educativas (pp. 23-38). Recuperado de http://www.oei.es/pdf2/aportes_educacion_no_formaluruguay.pdf

Chacón-Ortiz, M. (2015). El proceso de evaluación en educación no formal. Un camino para su construcción. Educare, 19(2), 21-35.

http://dx.doi.org/10.15359/ree.19-2.2

Código de la Niñez y la Adolescencia (2008). Gobierno de Costa Rica. Recuperado de http://www.oas.org/dil/esp/codigo_ninez_adolescencia_costa_rica.pdf

Dave, R., Ranaweera, A. y Sutton, P. (1990). Educación no formal. Chasqui. Revista Latinoamericana de Comunicación, (36), 35-41.

http://dx.doi.org/10.16921/chasqui.v0i36.2017 
García Arieto, L., Ruiz Corbella, M. y García Blanco, M. (2009). Claves para la educación. Actores, agentes y escenarios en la sociedad actual. Recuperado de https://www.researchgate.net/publication/235791982_Claves_para_la_Educa cion_Actores_agentes_y_escenarios_en_la_sociedad_actual

Grandi, J. (2009). Presentación a cargo de la UNESCO. En M. Morales (Comp.), Aportes para la elaboración de propuestas de políticas educativas (pp. 17-20). Recuperado de http://www.oei.es/pdf2/aportes_educacion_no_formaluruguay.pdf

Lamata, R. y Domínguez, R. (2003). La construcción de procesos formativos en educación no formal. Madrid, España: Narcea.

Luján Ferrer, M. (2010). La administración de la educación no formal aplicada a las organizaciones sociales. Aproximaciones teórico-prácticas. Educación, 34(1), 101-118. Recuperado de https://revistas.ucr.ac.cr/index.php/educacion/article/download/500/505

Morales, M. (2009). Aportes para la elaboración de propuestas de políticas educativas. Recuperado de http://www.oei.es/pdf2/aportes_educacion_no_formaluruguay.pdf

Pastor, M. (2001). Orígenes y evolución del concepto de educación no formal. Revista Española de Pedagogía, (220), 525-544. Recuperado de https://dialnet.unirioja.es/descarga/articulo/23701.pdf

Rodríguez. D. (2009). Contenidos en Educación No Formal. En M. Morales (Comp.), Aportes para la Elaboración de Propuestas de Políticas Educativas (pp. 153-161). Recuperado de http://www.oei.es/pdf2/aportes_educacion_no_formaluruguay.pdf

Torres Martín, C. y Pareja Fernández de la Reguera, J. A. (2007). La educación no formal y diferenciada. Fundamentos didácticos y organizativos. Madrid, España: CCS. 
Torres, R. (2009). Evaluación en Educación No Formal. En M. Morales (Comp.), Aportes para la elaboración de propuestas de políticas educativas (pp. 163171). Recuperado de

http://www.oei.es/pdf2/aportes_educacion_no_formaluruguay.pdf

Tünnermann Bernheim, C. (2010). La educación permanente y su impacto en la educación superior. Nuevos documentos sobre Educación Superior. Estudios e Investigaciones, (11). UNESCO. Recuperado de https://unesdoc.unesco.org/ark:/48223/pf0000120441

Turbay, C. (2000). El derecho a la educación. Desde el marco de la protección integral de los derechos a la niñez y de la política educativa. Santafé de Bogotá, Colombia: Fundación Antonio Restrepo Barco y UNICEF Colombia.

Recuperado de https://www.unicef.org/colombia/pdf/educacion.pdf 